\title{
Molecular Effect of PVP on The Release Property of Carvedilol Solid Dispersion
}

\author{
Myeong Jun Oh, Jung Bo Shim, Eun Yong Lee, Hanna Yoo, Won Hyung Cho, \\ Dong Kyun Lim, Dongwon Lee $^{\dagger}$ and Gilson Khang ${ }^{\dagger}$ \\ ${ }^{1}$ Department of BIN Fusion Technology \& Department of Polymer Nano Science Technology, \\ World Class Univ, Chonbuk Nat'l Univ, 664-14, Dukjin, Jeonju, 561-756, Korea \\ (Received March 25, $2011 \cdot$ Revised May 24, $2011 \cdot$ Accepted June 9, 2011)
}

\begin{abstract}
This study aimed to confirm the effect of molecular weight (MW) in solid dispersion of carvedilol with polyvinylpyrrolidone (PVP) of various MW. Solid dispersion of carvedilol with PVP was prepared by spray-drying method. Scanning electron microscopy (SEM) was used to analyze the surface of solid dispersion samples. Differential scanning calorimetry (DSC) and X-ray diffraction (XRD) were used to analyze the crystalline of solid dispersion. Fourier transform infrared spectroscopy (FT-IR) was used to analyze the change of chemical structure characteristic of solid dispersion. DSC and XRD show that drug crystalline was changed. FT-IR revealed that chemical structure of solid dispersion comparing the chemical structure of drug was changed. The dissolution studies of solid dispersion presented at simulated gastric juice $(\mathrm{pH}$ 1.2). The dissolution rate of solid dispersion was dramatically enhanced than pure drug and the MW of PVP has an effect on the release property of carvedilol in solid dispersion. In conclusion, the present study has confirmed the effect of MW of PVP on release property of solid dispersion formulation of carvedilol with PVP.
\end{abstract}

Key words - Carvedilol, PVP, Molecular weight, Solid dispersion, Spray-drying

Carvedilol (1-(9H-carbazol-4-yloxy)-3-[[2-(2-methoxy-phenoxy)ethyl]amino] -2-propanol) is a novel, multiple-action cardiovascular drug that is currently approved not only for the treatment of hypertension but also treat the chronic stable angina in many countries. Carvedilol (Figure 1-a) has reduced peripheral resistance and maintaines peripheral blood flow and renal blood rate gave an excellent level of blood pressure lowering produced by carvedilol, results from beta-alrenoceptor blockade, vasodilation and alpha 1-adrenoceptor blockade (Robert et al., 1992; Tanwar et al., 2007; Dunn et al., 1997). Carvedilol was a poor water-soluble oral antihypertensive agent with some problems of variable bioavailability and bioinequivalence which was related to its poor water-solubility. It has available as tablets for oral administration and has 25 30\% of bioavailability, $6 \sim 10$ hours of biological half-life and excretion through $16 \%$ of urine and $60 \%$ of digestive tract after metabolic reaction in the liver. It reaches its highest plasma level within 1.47 hours after dosage (Loftsson et al., 2008). However, the solubility of carvedilol in aqueous solutions with $\mathrm{pH}$ range from 1 to 4 was limited due to its protonation.

It is desirable to improve the solubility of insoluble drugs and the methods such as decreasing the particle size to increase

\footnotetext{
${ }^{\dagger}$ Corresponding Author :

Tel : +82-63-270-2336, E-mail : dlee@jbnu.ac.kr, gskhang@jbnu.ac.kr DOI : 10.4333/KPS.2011.41.3.179
}

the surface area, adding surfactant and using co-solvent to increase the solubility have been widely used. Self emulsifying, self micro emulsifying and the reduction of crystalline using hydrophilic polymers can also enhance the solubility of carvedilol efficiently. The most promising method for promoting dissolution might be the formation of solid dispersion (SD) in a proper carrier. Recently, the incorporation of drug into water-soluble polymer has been reported to result in an amorphous solid form with ease process and the dissolution of drug leading to improve its bioavailability (Ahn et al., 2004; Cao et al., 2003; Park et al., 2010).

SDs were generally prepared by either solvent method and the drug and carrier were dissolved in a solvent followed by removal or by co-melting method. The drug-carrier mixtures were prepared by co-melting or cooling methods. The SD system where drug molecules are dispersed in the carrier such as PVP or PEG is an alternative useful method. The techniques for the enhancing dissolution rate of SDs have been proposed. Drugs molecularly dispersed in polymeric carriers can provided reduction of particle size and increased the surface area. The techniques focused on the change their crystalline form to the amorphous form. However, the drug solubility may be increased by surrounding hydrophilic carriers. Because of the low dissolution rate of carvedilol, the techniques have been applied to improve the proper oral administration and enhance the drug release rate (Kim et al., 2010; Khang et al., 2001; Cho 
<smiles>CC(=O)c1ccccc1OCCNCC(O)COc1cccc2[nH]c3ccccc3c12</smiles>

(a)

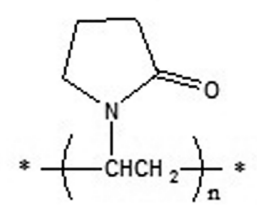

(b)

Figure 1. Chemical structures of (a) Carvedilol and (b) PVP.

et al., 2010). Hydrophilic polymers such as hydroxypropylcellulose (HPC), hydroxypropylmethylcellulose (HPMC), polyvinylpyrrolidone (PVP) and cellulose acetate have been widely investigated for SDs. PVP (Figure 1-b) is amongst the most frequently investigated hydrophilic polymeric carriers. It has been proposed that the extent of interaction with various organic molecular in solvent is related to crystalline suppression. (Jeong et al., 2002; Jeong et al., 2000). Molecules of PVP have arranged of 2,500 3,000,000 $\mathrm{g} / \mathrm{mole}$ (Waling, 1994) and it had been reported that the dissolution rate of SD could be improve the hydrophilic property of PVP. The PVP polymer chain length according to the molecular weight has been reported that it affects solubility of drugs in the SD. Thus, we studied controlled drug release behavior using PVP which has various $\mathrm{K}$ value.

The aim of this study, we would observe the release behavior of carvedilol in $\mathrm{pH}$ 1.2. The SD prepared with the varying molecular weight of PVP by spray-drying method. The surface of SD particles was analyzed by scanning electron microscope (SEM). The crystallinity of carvedilol was confirmed by differential scanning calorimetry (DSC) and X-ray powder diffraction (XRD). Chemical combination of both carvedilol and PVP was characterized by Fourier transform infrared spectroscopy (FT-IR). The effects of molecular weight of PVP on its dissolution properties were investigated.

\section{Materials and Methods}

\section{Materials}

Carvedilol (Aurobindo Pharma Limited, Andhra Pradesh, India) was used by the model drug in this study. Carrier using a preparation of SD was PVP (BASF, Ludwigshafen, Rheinland-Pfalz, Germany) obtained from BASF. All other materials and reagents were of HPLC grade and used as received
Table I. Conditions of spray-drying

\begin{tabular}{cc}
\hline \hline Parameter & Setting \\
\hline Inlet Temperature & $130^{\circ} \mathrm{C}$ \\
Outlet Temperature & $70 \pm 5^{\circ} \mathrm{C}$ \\
Atomizing & $10 \times 5 \mathrm{kPa}$ \\
Flow Rate & $0.45 \mathrm{~m}^{3} / \mathrm{min}$ \\
Pump Speed & $3.0 \mathrm{~mL} / \mathrm{min}$ \\
\hline
\end{tabular}

Table II. Preparation conditions and inclusion efficiency of SDs (Weight ratio)

\begin{tabular}{cccccc}
\hline $\begin{array}{c}\text { Batch } \\
\text { No. }\end{array}$ & Drug & $\begin{array}{c}\text { PVP K-17 } \\
(\text { Mw:8,000) }\end{array}$ & $\begin{array}{c}\text { PVP K-25 } \\
(\text { Mw:30,000) }\end{array}$ & $\begin{array}{c}\text { PVP K-30 } \\
(\text { Mw:50,000) }\end{array}$ & $\begin{array}{c}\text { Inclusion } \\
\text { efficiency } \\
(\%)\end{array}$ \\
\hline 1 & 1 & 1 & & & 50.76 \\
2 & 1 & 3 & & & 73.70 \\
3 & 1 & & 1 & & 48.60 \\
4 & 1 & & 3 & & 76.25 \\
5 & 1 & & & 1 & 48.32 \\
6 & 1 & & & 3 & 76.49 \\
\hline
\end{tabular}

without further purification.

\section{Preparation of solid dispersion}

Carvedilol and PVP were taken in ratio of Table II and dissolved in mixture of methanol and acetone 1:1 (v/v\%) ratio. This solution was stirred at 30 minutes and prepared by SD using a spray-dryer (Spray Dryer SD-1000, Eyela, Tokyo, Japan). The condition of spray-dryer listed on Table I. The prepared powder was stored in desiccator until further use.

\section{Inclusion efficiency}

The SD was dissolved in $20 \mathrm{~mL}$ methanol and then extracted sample was filtered by $0.45 \mu \mathrm{m}$ PTFE filter (Tokyo Roshi Kaisha, Ltd., Tokyo, Japan) and measured by HPLC at $240 \mathrm{~nm}$ with a UV detector. The inclusion efficiency was defined as the ratio of actual and original amount of carvedilol in SD. The calculation equation is followed (Cho et al., 2010; Eom et al., 2010).

Inclusion efficiency $(\%)=$

$$
\frac{\text { Actual amount of drug in SD }}{\text { Theoretical amount of drug in SD }} \times 100
$$

\section{Solubility measurements of carvedilol}

An excess amount of carvedilol was added to $10 \mathrm{ml}$ of aqueous solutions having $1 \mathrm{mg}$ of PVP K-17, PVP K-25 or PVP K30. The samples were stirred for $48 \mathrm{~h}$ at room temperature. Subsequently, the suspensions were filtered through $0.45 \mu \mathrm{m}$ 
PTFE filter (Tokyo Roshi Kaisha, Ltd., Tokyo, Japan) and the diluted solutions were analyzed by using HPLC at $240 \mathrm{~nm}$ with UV detector.

Morphological observation of solid dispersion using scanning electron microscopy (SEM)

The particles of SD were analyzed by SEM. The samples were then sputter-coated with Pt for $10 \mathrm{~min}$ at $30 \mathrm{~mA}$. SEM was taken by using a LV-SEM (S-3000N, Hitachi, Tokyo, Japan).

Crystallinity studies by X-ray diffraction and Differential scanning calorimetry (DSC)

XRD patterns were recorded on a MAX 2500 X-ray diffractometer (Rigaku, Tokyo, Japan). The pattern range is $5 \sim 40^{\circ}$. Standard runs a $40 \mathrm{kV}, 30 \mathrm{~mA}$, and $0.5^{\circ} / \mathrm{min}$ scanning rate (Henderson et al., 2008). DSC thermograms were recorded on a DSC Q20 (TA Instruments-Waters LLC, Newcastle, Delaware, USA). The samples were sealed in aluminum pans and heated rate $10^{\circ} \mathrm{C} / \mathrm{min}$ over a temperature range $30 \sim 150^{\circ} \mathrm{C}$ (Piera et al., 2007).

Investigation of chemical structures by fourier transform infrared spectroscopy (FT-IR)

Infrared spectra were recorded on a spectrum GX (Perkin Elmer, Waltham, Massachusetts, USA). A spectra range is $400 \sim 4000 \mathrm{~cm}^{-1}$. A KBr disc method was used with $1 \%$ (w/w) sample loading.

\section{Dissolution studies}

Dissolution studies were performed with capsule containing $60 \mathrm{mg}$ SDs in $900 \mathrm{~mL}$ of simulated gastric juice (pH 1.2) with paddle stirring at a speed of $50 \mathrm{rpm}$ at $37 \pm 0.5^{\circ} \mathrm{C}$. The dissolution test was used to DST-610 (Labfine Sci Instr, Korea). The release of carvedilol was measured by withdrawing samples at regular intervals and filtered through a pore size $0.45 \mu \mathrm{m}$ membrane filter (Tokyo Roshi Kaisha, Ltd, Japan). The samples with drawn were replaced with fresh medium maintained at the same temperature.

\section{HPLC analysis}

The chromatographic analyses were performed on HPLC system consisting of an NS-4000 HPLC system (Futecs, Daejeon, Korea) linked to an NS-6000 Autosampler (Futecs, Daejeon, Korea). The mobile phase was $650 \mathrm{ml} \mathrm{DW}$ with $\mathrm{KH}_{2} \mathrm{PO}_{4}$ $1.77 \mathrm{~g}$, adjustment to $\mathrm{pH} 2.0$ with phosphoric acid, and mixing with acetonitrile to a 50:50 (v/v\%) ratio. The mobile phase was used after removal of residual gas by ultrasonication. Carvedilol was detected by absorbance at $240 \mathrm{~nm}$, and confirmed using Intersil OSD-3 $\mathrm{C}_{18}$ (4.6150 mm, $5 \mu \mathrm{m}$, GL Science Inc, Torrance, California, USA). Each injection amount was set to $20 \mu \mathrm{L}$ and the flow rate at $1.5 \mathrm{~mL} / \mathrm{min}$.

\section{Results and Discussion}

\section{Preparation of solid dispersion and inclusion efficiency}

Table II listed that the formulation of carvedilol SD and included efficiency of drug in SD. The inclusion efficiency of drug in SD was changed by polymer ratio in SD. According to increasing polymer ratio in $\mathrm{SD}$, inclusion efficiency was increased. These results were caused by polymers that were decomposed by high temperature and pressure in spray-drying process when SD has lower polymer content ratio (Lee et al., 2010; Ohya et al., 1993; Lee et al., 2008). On the other hand, increasing polymer content ratio in SD showed high inclusion efficiency because of their effectively interaction between drug and polymer (Park et al., 2008; Chung et al., 2001).

\section{Solubility studies}

Figure 2 shows the solubility of carvedilol on the equally 1 $\%$ concentration of PVP K-17, K-25 and K-30 in distilled water (DW) at room temperature. The solubility of carvedilol in polymer solution was higher than solubility of carvedilol in DW. The result suggested that PVP increased the solubility of carvedilol. In addition, the increase in solubility of carvedilol by PVP may probably be due to the formation of soluble complexes between water-soluble polymeric carrier and poorly soluble drug. Thus, PVP was selected for formulation of SDs because of its better solubility of carvedilol in its aqueous solution.

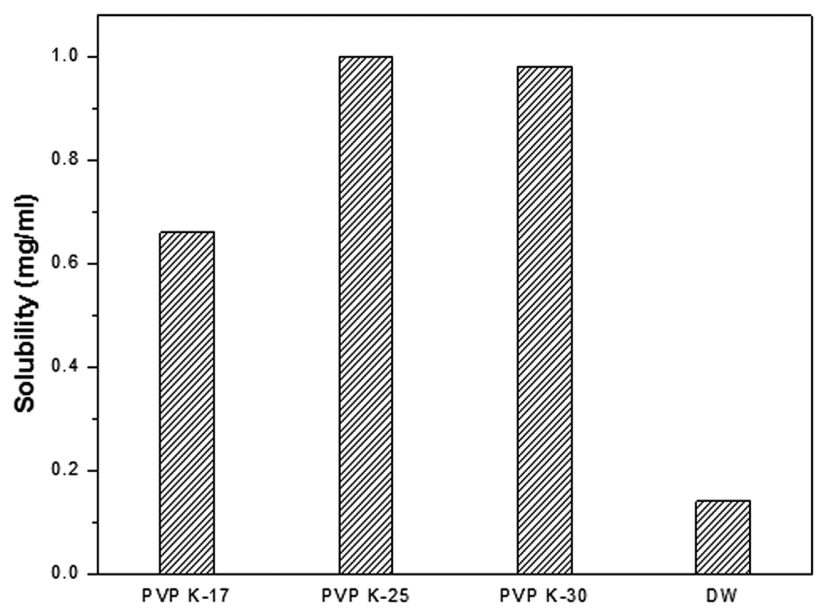

Figure 2. Solubility of carvedilol in the various K values of PVP solution. 

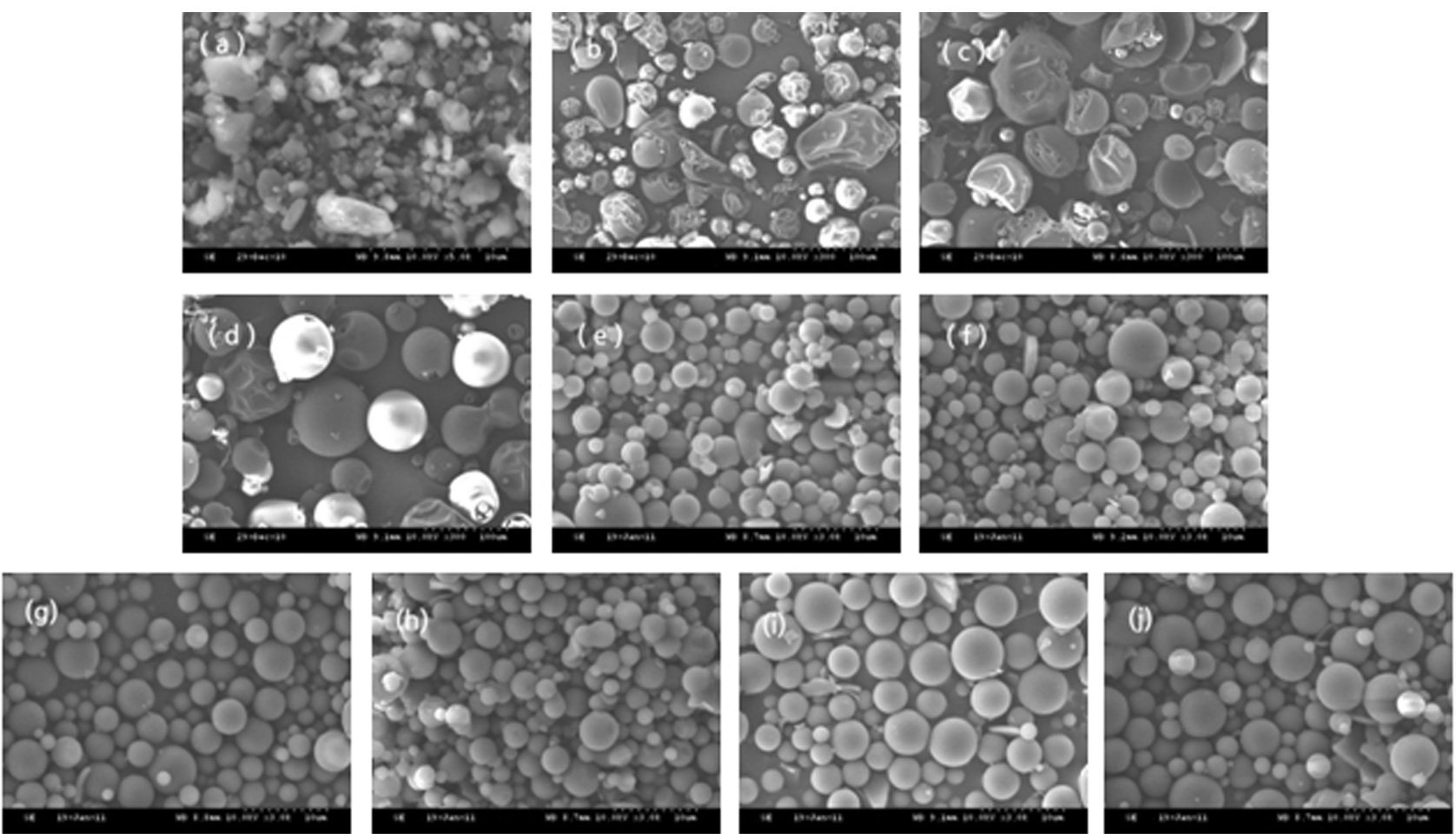

Figure 3. SEM morphology images of SD. (a) Carvedilol, (b) PVP K-17, (c) PVP K-25, (d) PVP K-30, (e) SD (carvedilol:PVP K-17=1:1), (f) SD (carvedilol:PVP K-17=1:3), (g) SD (carvedilol:PVP K-25=1:1), (h) SD (carvedilol:PVP K-25=1:3), (i) SD (carvedilol:PVP K$30=1: 1$ ), (j) SD (carvedilol:PVP K-30=1:3).

\section{Morphology observation of solid dispersion}

SEM photographs of pure carvedilol, PVP and SD samples were taken to study their morphology (Figure 3). As shown in Figure 3, the particle size of PVP was larger than that of SD, all of SDs showed sphere form and were almost equaled in particle size. These results suggested that the sphere form was made by the process of spray-drying in high temperature and pressure or interaction between drug and polymer.

\section{Crystalline studies by XRD and DSC}

The XRD of the carvedilol, PVP and the SD are showed in Figure 4. Characteristic peaks of carvedilol displayed a broad range. As a result, it confirmed that carvedilol was crystalline drug. However, the characteristic peak of carvedilol was not showed in SD. This results suggested that crystallinity of carvedilol was changed. Figure 5 showed DSC analysis of carvedilol, PVP and SD. The DSC curve of carvedilol showed a melting point peak at $120^{\circ} \mathrm{C}$. On the other hand, melting point peak was not showed in SD. It was confirmed that crystallinity of carvedilol changed to the amorphous state as the results of XRD analysis. The reduced crystallinity of the drug could be one of the mechanisms responsible for improved dissolution.

In the results of DSC and XRD analysis, carvedilol was

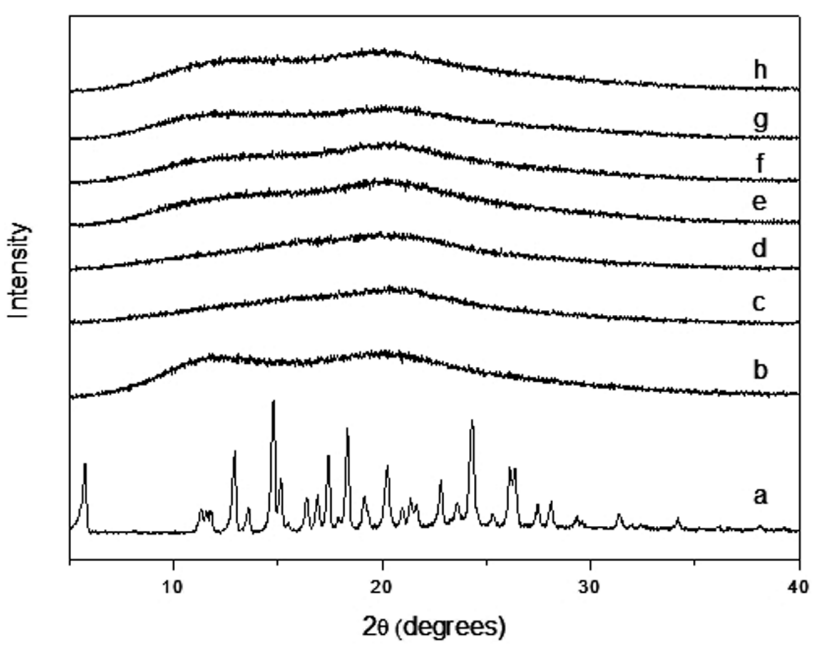

Figure 4. X-ray diffraction patterns of (a) carvedilol, (b) PVP, (c) SD (carvedilol:PVP K-17=1:1), (d) SD (carvedilol:PVP K-17=1:3), (e) SD (carvedilol:PVP K-25=1:1), (f) SD (carvedilol:PVP K25=1:3), (g) SD (carvedilol:PVP K-30=1:1), (h) SD (carvedilol:PVP $\mathrm{K}-30=1: 3)$.

crystallinity drug. However, prepared SD did not showed original crystallinity and melting point peak of carvedilol in DSC and XRD analysis. Thus, carvedilol in SD was might be in an amorphous state in the SD because of high temperature and pressure in spray-drying process (Dahlberg et al., 2010). 


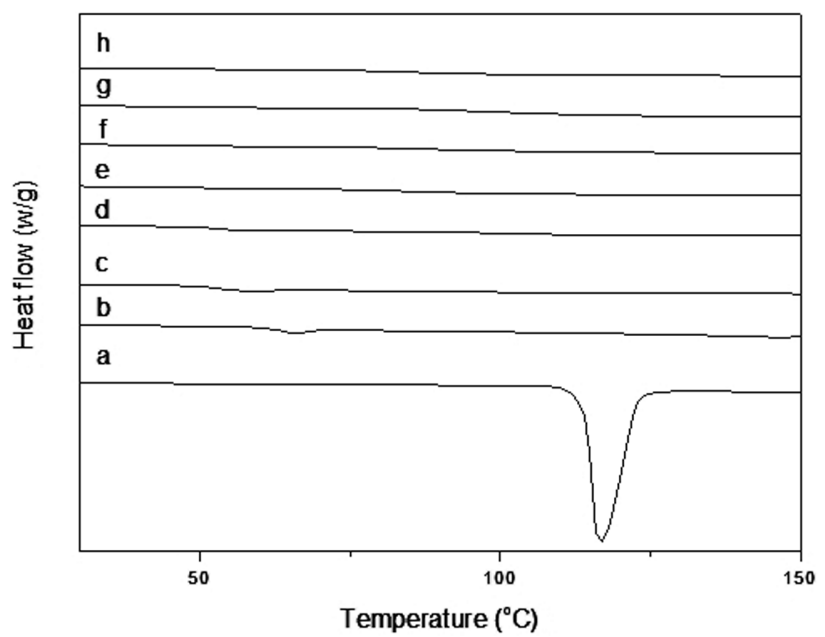

Figure 5. DSC thermograms of SD of carvedilol with PVP at various weight ratios. (a) carvedilol, (b) PVP, (c) SD (carvedilol:PVP K-17=1:1), (d) SD (carvedilol:PVP K-17=1:3), (e) SD (carvedilol: PVP K-25=1:1), (f) SD (carvedilol:PVP K-25=1:3), (g) SD (carvedilol: PVP K-30=1:1), (h) SD (carvedilol:PVP K-30=1:3).

\section{Investigation of chemical structures}

Figure 6 shows the results of FT-IR analysis that was to confirm the changed chemical structure due to interaction with both carvedilols. Carvedilol showed characteristic peaks between 3,300 and 3,500 $\mathrm{cm}^{-1}(\mathrm{~N}-\mathrm{H}$ stretching vibration peaks of amine group) and PVP showed characteristic peaks between 1,500 and $1,700 \mathrm{~cm}^{-1}(\mathrm{C}=\mathrm{O}$ stretching vibration peaks of carbonyl group) in all SD. When it was compared the analyzed results of FT-IR of SD with the analyzed results of carvedilol, PVP, N-H stretching vibration peaks of carvedilol was disappeard and $\mathrm{C}=\mathrm{O}$ stretching vibration peaks of PVP was increased markedly. This results were suggest that the hydrogen bond of carvedilol and PVP.

\section{Dissolution studies}

Figure 7 shows the dissolution profiles of pure drug and various SD formulations. As shown in Figure 7, all SD formulations achieved higher dissolution rate than pure drug. The SD of carvedilol with PVP K-17 in the weight ratio of $1: 1$ showed approximately $90 \%$ dissolution rate. However, the SD of carvedilol with PVP K-25 or K-30 in the weight ratio of 1:1 showed approximately $68 \%$ and $48 \%$ dissolution rate respectively. The SDs of carvedilol with PVP in the weight ratio of 1:3 showed $100 \%$ dissolution rate in all. These datas were in conformity with solubility data. Moreover, the fast dissolution behavior of the SD may be due to the amorphous state of carvedilol in SD. Also the initial dissolution behavior was difference among the SDs of carvedilol with PVP in the weight ratio of $1: 3$. These results suggested that the relatively high

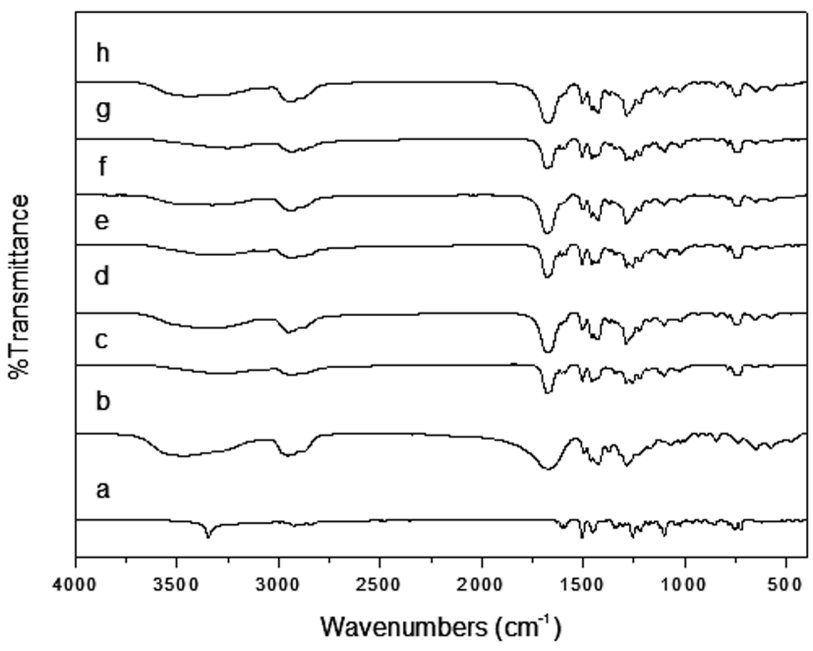

Figure 6. Fourier transform infrared spectroscopy spectras of SD. (a) carvedilol, (b) PVP, (c) SD (carvedilol:PVP K-17=1:1), (d) SD (carvedilol:PVP K-17=1:3), (e) SD (carvedilol:PVP K-25=1:1), (f) SD (carvedilol:PVP K-25=1:3), (g) SD (carvedilol:PVP K-30=1:1), (h) SD (carvedilol:PVP K-30=1:3).

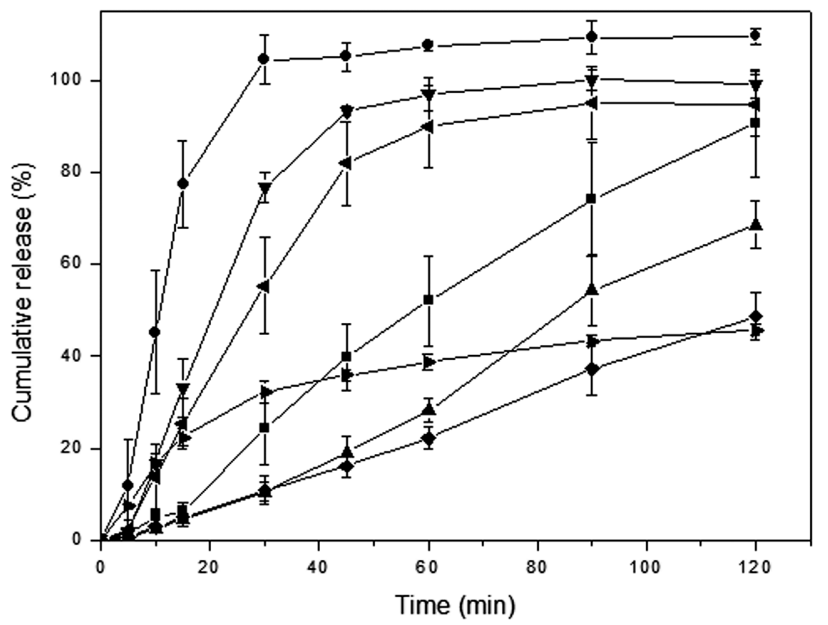

Figure 7. Dissolution profiles in simulated gastric juice $(\mathrm{pH} 1.2)$ at $50 \mathrm{rpm}, 37.5 \pm 0.5^{\circ} \mathrm{C}$ for $2 \mathrm{hrs}(\mathrm{n}=3)$. ( $) \mathrm{SD}$ of carvedilol:PVP K$17=1: 1$, (О) SD of carvedilol:PVP K-17=1:3, (⿻) SD of carvedilol:PVP K-25=1:1, ( $)$ SD of carvedilol:PVP K-25=1:3, ( ) SD of carvedilol:PVP K-30=1:1, ( 4 ) SD of carvedilol:PVP K$30=1: 3$, ( $\gg$ ) pure drug.

molecular weight of PVP K-25, K-30 had an effect on release property of carvedilol in SD and the viscosity of the polymer delayed the initial dissolution rate of SD.

\section{Conclusions}

In this study, SD was prepared by using spray-drying method to enhance the dissolution rate of carvedilol and to investigate the molecular weight effect of PVP. This study has demonstrated that molecular weight of PVP has an effect on the 
release property of carvedilol. It means that the polymer chain length according to the molecular weight has affected solubility of drug in the SD. Furthermore, the dissolution rate was dramatically higher than that of pure carvedilol due to the crystallinity of carvedilol in SD was changed to amorphous form.

Based on the results, SD has great potential for possibility of pharmacological oral dosage form because it has controlled release behavior of carvedilol in this system. Furthermore, we observed that the transformation of carvedilol form from the crystalline to amorphous state and it remarkably improved the dissolution rate.

\section{Acknowledgements}

This study was supported by WCU (R31-20029).

\section{References}

Ahn, Y.S., Lee, H.Y., Hong, K.D., Jung, S.B., Cho, S.H., Rhee, J.M., Lee, H.B., Khang, G., 2004. Effect of types and mixing ratios of water-soluble polymers on in vitro release profile of SD for acyclovir. J. Kor. Pharm. Sci. 34, 289-297.

Cao, Q.R., Kim, T.W., Choi, C.Y., Kwon, K.A., Lee, B.J., 2003. Preparation and dissolution of polyvinylpyrrolidone(PVP)based SD systems containing solubilizers. J. Kor. Pharm. Sci. $33,7-14$.

Cho, W.H., Lee, Y.H., Oh, M.J., Lee, D., Khang, G., 2010. Solubility characterization and improved dissolution of pranlukast with the surfactant. Int J. Tissue Reg. 1, 37-45.

Cho, W.H., Lee, Y.H., Song, B.J., Yoo, S.C., Lee, D.W., Khang, G., 2010. Improvement of pranlukast dissolution by nano SD. Int J. Tissue Reg. 1, 74-80.

Chung, T.S., Tun, C.M., Pramoda, K.P., Wang, R., 2001. Novel hollow fiber membranes with defined unit-step morphological change. J. Membr Sci. 193. 123-128.

Dahlberg, C., Fureby, A.M., Schuleit, M., Furó, I., 2010. Polymerdrug interactions and wetting of SDs. Eur J Pharm Sci. 39, 125-133.

Dunn, C.J., Lea, A.P., Wagstaff, A.J., 1997. Carvedilol: a reappraisal of its pharmacological $\beta$-blockers in left ventricular dysfunction and heart failure. Drugs. 54, 161-169.

Eom, S., Yoo, S.C., Song, B.J., Kim, Y.K., Park, H.J., Seong, K.Y., Song, Y.S., Lee, D.W., Khang, G., 2010. Effect of preparation conditions on release behavior of raloxifene HCL-loaded PLGA microspheres. Int J Tissue Reg. 1, 28-36.

Henderson, E.J., Hessel, C.M., Veinot, JG.C., 2008. Synthesis and photoluminescent properties of size-controlled germanium nanocrystals from phenyl trichlorogermane-derived polymers. J. Am Chem Soc. 130, 3624-3632.
Jeong, J.K., Khang, G., Rhee, J.M., Shin, H.C., Lee, H.B., 2000. Effect of molecular weight and mixture ratio of PVP on improving the bioavailability of ipriflavone. J. Korean Pharm. Sci. 30(4), 235-239.

Jeong, J.K., Kim, J.H., Khang, G., Rhee, J.M., Lee, H.B., 2002. The preparation and characterization of SD of ipriflavone with PVP. J. Korean Pharm Sci. 32(3), 173-179.

Khang, G., Jeong, J.K., Rhee, J.M., Lee, J.S., Lee, H.B., 2001. Effect of hydrophillic polymers on SD of ipriflavone to improve bioavailability, Macromol. Chem. Symp. 14, 123132.

Kim, Y.K., Lim, D.K., Lee, S.K., Eom, S., Lee, E.Y., Kim, S.J., Lee, D., Khang, G., 2010. Characterization and enhanced dissolution rate of nateglinide SD by using polyvinylacetal diethylaminoacetate. Int J. Tissue Reg. 1, 101-107.

Lee, E.Y., Oh, M.J., Kim, Y.K., Eom, S., Park, H.J., Seong, K.Y., Kim, S.J., Jo, E.H., Lee, D.W., Khang, G., 2010. Controlled release of rosiglitazone maleate ny $\mathrm{SD}$ with cellulose acetate. Int J. Tissue Reg. 1, 88-93.

Lee, J.H., Ku, J., Park, J.S., Park, J.H., Ahn, S.I., Mo, J.H., Kim, Y.T., Rhee, J.M., Lee, H.B., Khang, G., 2008. Improved dissolution and Characterization of solid dispersed atorvastatin calcium. J. Kor. Pharm. Sci. 38(2), 111-117.

Loftsson, T., Vogensen, SB., Desbos, C., Jansook, P., 2008. Carvedilol: solubilization and cyclodextrin complexation: a technical note. AAPS Pharm Sci Tech. 9, 425-430.

Ohya, H., Jicsh, H., Nrgish, Y., 1993. Studies on distribution and reverse-osmosis properties of cellulose-acetate derivatives for organic-compounds. J. Membrane Sci. 85(1), 1-11.

Park, J.S., Oh, J.M., Kim, Y.T., Lee, J.H., Kim, M.J., Mo, J.H., Kim, M.S., Lee, H.B., Khang, G., 2008. Release behavior from sibutramine microspheres using spray-dryer. Polymer(Korea). 32(3), 193-198.

Park, Y.J., Oh, D.H., Yan, Y.D., Seo, Y.G., Lee, S.N., Choi, H.G., Yong, C.S., 2010. Surface-attached SD. J Pharma Investi. 40, 97-102.

Piera, D.M., Roberta, C. Christine, B., Roberto, G., Etienne. J., Admir, M., Pascal, O., Sante, M., 2007. Characterization and compaction behaviour of nimesulide crystal forms, Int $\mathrm{J}$. Pharm. 342. 137-144.

Robert, R., Ruffolo, R.R., Boyle, DA., Brooks, D.P., Feuerstein, G.Z., Venuti, R.P., Lukas, M.A., Paste, G., 1992. Carvedilol: a novel cardiovascular drug with multiple actions, Cardiovasc Drug Rev. 10, 127-157.

Tanwar, Y.S., Chauhan, C.S., Sharma, A., 2007. Development and evaluation of carvedilol transdermal patches. Acta Pharm. 57, 151-159.

Waling, W.D., 1994. "Povidone", in "Handbook of Pharmaceutical Excipients", A Wade and PJ Weller, EDS, 392, American Pharmaceutical Association/The Pharmaceutical Press, Washington, DC/London, 392. 